\title{
Pyranometer offsets triggered by ambient meteorology: insights from laboratory and field experiments
}

\author{
Sandro M. Oswald ${ }^{1,2,3}$, Helga Pietsch ${ }^{2}$, Dietmar J. Baumgartner ${ }^{4}$, Philipp Weihs ${ }^{3}$, and Harald E. Rieder ${ }^{1,2,5}$ \\ ${ }^{1}$ Wegener Center for Climate and Global Change, Graz, Austria \\ ${ }^{2}$ Institute for Geophysics, Astrophysics and Meteorology, Institute of Physics, Graz, Austria \\ ${ }^{3}$ Institute of Meteorology, University of Natural Resources and Life Sciences (BOKU), Vienna, Austria \\ ${ }^{4}$ Kanzelhöhe Observatory for Solar and Environmental Research, Graz, Austria \\ ${ }^{5}$ Austrian Polar Research Institute, Vienna, Austria \\ Correspondence to: Sandro M. Oswald (sandro.oswald@boku.ac.at)
}

Received: 19 October 2016 - Discussion started: 30 November 2016

Revised: 24 February 2017 - Accepted: 26 February 2017 - Published: 21 March 2017

\begin{abstract}
This study investigates the effects of ambient meteorology on the accuracy of radiation $(R)$ measurements performed with pyranometers contained in various heating and ventilation systems (HV-systems). It focuses particularly on instrument offsets observed following precipitation events. To quantify pyranometer responses to precipitation, a series of controlled laboratory experiments as well as two targeted field campaigns were performed in 2016. The results indicate that precipitation (as simulated by spray tests or observed under ambient conditions) significantly affects the thermal environment of the instruments and thus their stability. Statistical analyses of laboratory experiments showed that precipitation triggers zero offsets of $-4 \mathrm{Wm}^{-2}$ or more, independent of the HV-system. Similar offsets were observed in field experiments under ambient environmental conditions, indicating a clear exceedance of BSRN (Baseline Surface Radiation Network) targets following precipitation events. All pyranometers required substantial time to return to their initial signal states after the simulated precipitation events. Therefore, for BSRN-class measurements, the recommendation would be to flag the radiation measurements during a natural precipitation event and $90 \mathrm{~min}$ after it in nighttime conditions. Further daytime experiments show pyranometer offsets of $50 \mathrm{~W} \mathrm{~m}^{-2}$ or more in comparison to the reference system. As they show a substantially faster recovery, the recommendation would be to flag the radiation measurements within a natural precipitation event and $10 \mathrm{~min}$ after it in daytime conditions.
\end{abstract}

\section{Introduction}

Earth's climate is largely determined by the global energy balance (Wild et al., 2012). Therefore, a precise knowledge of the surface energy budget, which includes the solar and terrestrial radiation fluxes, is essential for understanding the Earth's planetary circulation and climate system (Ramanathan, 1987; Augustine and Dutton, 2013; Wild et al., 2014).

In situ measurements of solar radiation on the Earth's surface, more precisely global radiation which is the sum of the direct and diffuse components, began in the 1920s but became more widespread with the advent of thermopile pyranometers and through initiatives of the International Geophysical Year, 1957-1958 (Wild, 2009). Around the turn of the century a series of studies (Dutton et al., 1991; Gilgen et al., 1998; Ohmura et al., 1998; Stanhill, 2005; Liepert, 2002) reported negative trends of global radiation based on in situ measurements, a phenomenon commonly referred to as "global dimming" (Wild, 2005, 2009). Average trends of -6 to $-9 \mathrm{~W} \mathrm{~m}^{-2}$ between $1960-1990$ have been reported in the literature (Wild, 2005), but estimates vary depending on location, record length, and time period considered (Wild et al., 2012). The previously observed negative trends were replaced by a widespread increase in surface solar radiation over the period 1990-2000, a phenomenon commonly referred to as "global brightening" (Wild, 2005).

The growing interest of the scientific community in surface radiation trends and limitations in the accuracy of his- 


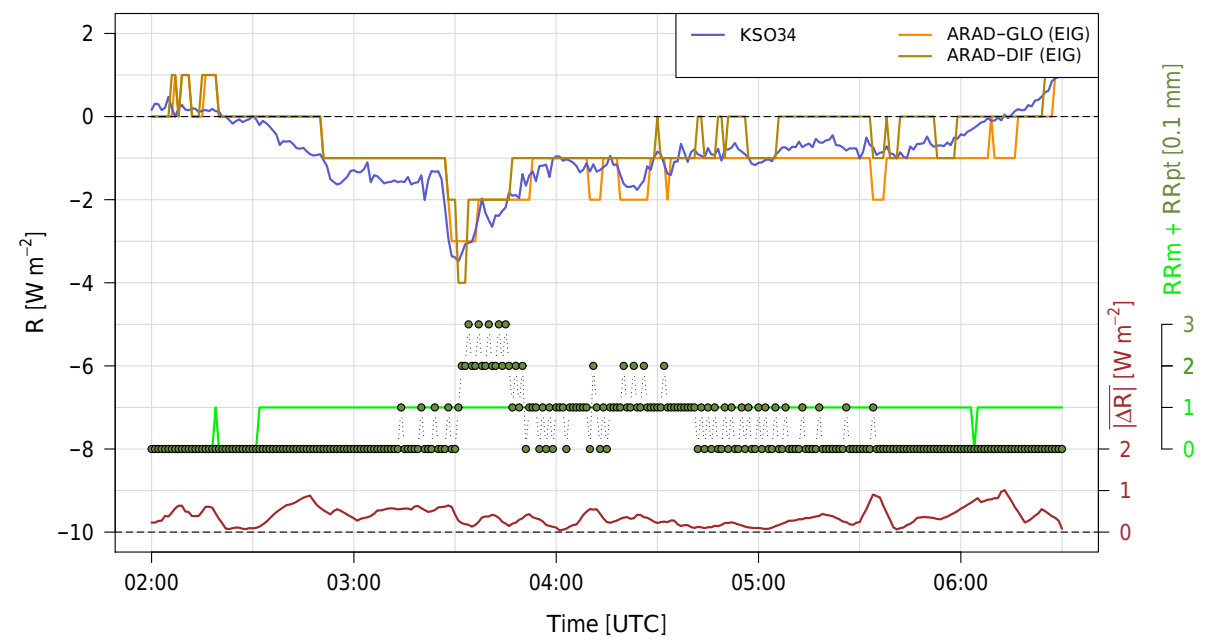

Figure 1. Natural event of a simultaneous decrease of radiation $(R)$ measured with three CMP21 pyranometers in various heating and ventilation systems at the Graz University ARAD site on 10 February 2016. System acronyms represent measurements of global (ARADGLO) and diffuse (ARAD-DIF) solar radiation at the ARAD platform (with CMP21 pyranometers contained in Eigenbrodt SBL 480, EIG, HV-systems) and global radiation measurements (KSO34) with an additional CMP21 pyranometer (contained in a KSO34 HV-system). The output of the precipitation sensor (RRm) and the ombrometer (RRpt) operated at the co-located meteorological Graz University station is shown along with the radiation measurements. Heavy precipitation started around 03:30 UTC.

toric records led in the early 1990s to the establishment of the Baseline Surface Radiation Network (BSRN) under the auspices of the World Climate Research Programme (Ohmura et al., 1998). BSRN sites are equipped with instruments of the highest accuracy and, to date, more than 50 anchor sites are operational around the globe. Besides BSRN, a series of national monitoring networks was established at this time operating at (or close to) BSRN standards.

One of these national monitoring networks is the so-called Austrian radiation monitoring network (ARAD), which was established in 2010 by a consortium of the Zentralanstalt für Meteorologie und Geodynamik (ZAMG), the University of Graz, the University of Innsbruck, and the University of Natural Resources and Life Sciences, Vienna (BOKU). ARAD aims to provide long-term monitoring of radiation budget components at highest accuracy and to capture the spatial patterns of radiation climate in Austria (Olefs et al., 2016). To date, the ARAD Network comprises one BSRN site (Sonnblick) and five additional sites (Kanzelhöhe Observatory, Graz University, Innsbruck, Klagenfurt, and Wien Hohe Warte). All ARAD sites are equipped with instrumentation according to BSRN standards (McArthur, 2005).

Despite BSRN-class equipment and regular instrument maintenance, radiation $(R)$ measurements are also influenced by meteorological conditions and instrumentation effects occasionally leading to so-called zero offsets (Kipp and Zonen, 2010).

Field measurements performed within the scope of ARAD indicate that such zero offsets are frequently triggered by precipitation events. However, to the knowledge of the authors, to date, no study has systematically investigated the influence of precipitation events on the accuracy of radiation measurements.

This study aims to close this gap by investigating the influence of precipitation events on the accuracy of radiation measurements under laboratory and field conditions. Three measurement campaigns, one under controlled laboratory conditions and two under ambient environmental conditions, were performed between January and May 2016. The campaign design was centered on zero offsets during nighttime conditions and on the influence of precipitation events on the accuracy of radiation measurements in the ARAD setup.

The particular interest in the influence of precipitation events stems from the regular observation of zero offsets (during nighttime conditions) following precipitation events within the ARAD network. Figure 1 illustrates such an event in the series of global and diffuse radiation measurements at the Graz University ARAD site.

\section{Methods and instrumentation}

During all campaigns, radiation measurements were performed with a series of CMP21 type pyranometers (manufactured by Kipp \& Zonen) which are routinely operated for the measurement of global and diffuse solar radiation at the majority of ARAD sites. The CMP21 pyranometer is composed of two quartz-glass domes, a black receiving area (sensing element), a thermal battery (thermopile), a thermalisation resistance and compensation element in the body, a thermistor for body temperature, and a drying cartridge. The black receiving area is connected with a passive sensing element called thermopile, which consists of 16 thermocouple junc- 
Table 1. Characteristics of the different heating and ventilation systems used in this study.

\begin{tabular}{llll}
\hline & System I & System II & System III \\
\hline Manufacturer & Eigenbrodt GmbH \& Co. KG & Observatory Kanzelhöhe & PMOD $^{1}$, World Radiation Center \\
Type & SBL 480 & KSO34 & PMOD-VHS \\
Acronym & EIG & KSO34 & DAV \\
Power & 24 VAC & 24 VAC & 24 VAC $^{2}$ \\
Ventilation & Continuous & Continuous & Continuous \\
Heating power & 10 W & $10 \mathrm{~W}$ & $10 \mathrm{~W}$ \\
Heating element & Discrete electrical resistor & Discrete electrical resistor & Circular heating element \\
\hline
\end{tabular}

${ }^{1}$ Physikalisch-Meteorologisches Observatorium Davos.

${ }^{2}$ Modified by ZAMG for use with $24 \mathrm{VAC}$; original PMOD configuration is for use with 12 VDC.

tion pairs connected electrically in series. The temperature of one of these thermocouple junctions, called an active or "hot" junction, increases with the absorption of solar radiation. A "cold" junction, fixed on the thermopile, is held at a constant temperature and serves as a reference for the hot junction. The differential temperature between the hot and cold junction produces an electromotive force directly proportional to the difference in temperature and is converted to an output voltage corresponding to the absorbed solar radiation. This process is referred to as the Seebeck effect. As every thermal battery has its own physical properties and structure, every radiometer has its specific and individual calibration factor. The black receiving area has a very rough surface structure with micro-cavities that effectively absorbs more than $97 \%$ of the shortwave radiation in a broad spectral range from 300 to $3000 \mathrm{~nm}$. CMP21 pyranometers are complying with the ISO 9060 standard and the guidelines of the World Meteorological Organization (Kipp and Zonen, 2010).

The body temperature of a pyranometer of the CMP series is measured by a thermistor (type YSI-44031, $10 \mathrm{k} \Omega$ @ $25^{\circ} \mathrm{C}$ ). This body temperature is directly proportional to the ambient air temperature whereby the possibility of the emergence of heat currents in the radiometer, causing a so-called "zero offset type B", has to be considered. Such zero offsets are specified by the manufacturer to occur following a $5 \mathrm{~K} \mathrm{~h}^{-1}$ change in ambient air temperature (Kipp and Zonen, 2010) over short time intervals.

Pyranometers used within the ARAD network are operated in different heating and ventilation systems. The set of ARAD HV-systems comprises the commercially available Eigenbrodt SBL 480 (EIG) and the PhysikalischMeteorologisches Observatorium (PMOD) VHS (DAV). In addition to these systems, a self-built $\mathrm{HV}$-system manufactured by the staff of the Kanzelhöhe Observatory (KSO34), was used during all campaigns. An overview of the characteristics of individual $\mathrm{HV}$-systems is provided in Table 1. Serial numbers of HV-systems and CMP21 pyranometers are provided for completeness in Table S1 in the Supplement to this article.
In addition to radiation measurements, standard meteorological observations of temperature, precipitation, relative humidity of air, and wind speed and direction were performed during the campaigns.

As our investigations focused mainly on the question of how precipitation events influence the accuracy of radiation measurements, a series of spray tests was performed during all campaigns. These spray tests were performed with an automated pumping system (designed and built by the staff of the Kanzelhöhe Observatory), applying 30 strokes of distilled water (approx. $3.4 \mathrm{~mL}$ ) from a vertical distance of $6 \mathrm{~cm}$ onto the pyranometer's glass dome. The spray system created very fine, homogeneous drizzle, producing small droplets onto the pyranometer dome, which quickly coagulated to larger drops. Such coagulation on pyranometer domes is also observed during stratiform and convective precipitation events.

CMP21 pyranometers were operated, in parallel, in different HV-systems during a laboratory campaign at the Kanzelhöhe Observatory (KSO, in January 2016) and during two field campaigns (one each in January and April to May 2016) at the measurement platform of the University of Graz in the direct vicinity of the ARAD site (see Fig. 2).

During the measurement campaigns, all CMP21 pyranometers were operated in the standard ARAD configuration for global radiation measurements at low- to mid-altitude sites (heating level $10 \mathrm{~W}$ ).

All measurement systems (i.e., pyranometers and HVsystems) were mounted in series on a stable aluminum jig, and a slide bar on the jig ensured seamless position changes of the electric motor pump for spray tests.

The first measurement campaign was performed between 6 and 17 January 2016 in the laboratory of the Kanzelhöhe Observatory. During this campaign, all pyranometers and HV-systems were operated under controlled ambient conditions at an approximately constant air temperature of $T_{\mathrm{a}} \approx 7^{\circ} \mathrm{C}$ and an approximately constant relative humidity of $\mathrm{RH} \approx 65 \%$. As we are particularly interested in zero offsets, pyranometers were operated in the dark. Figure 2a provides 


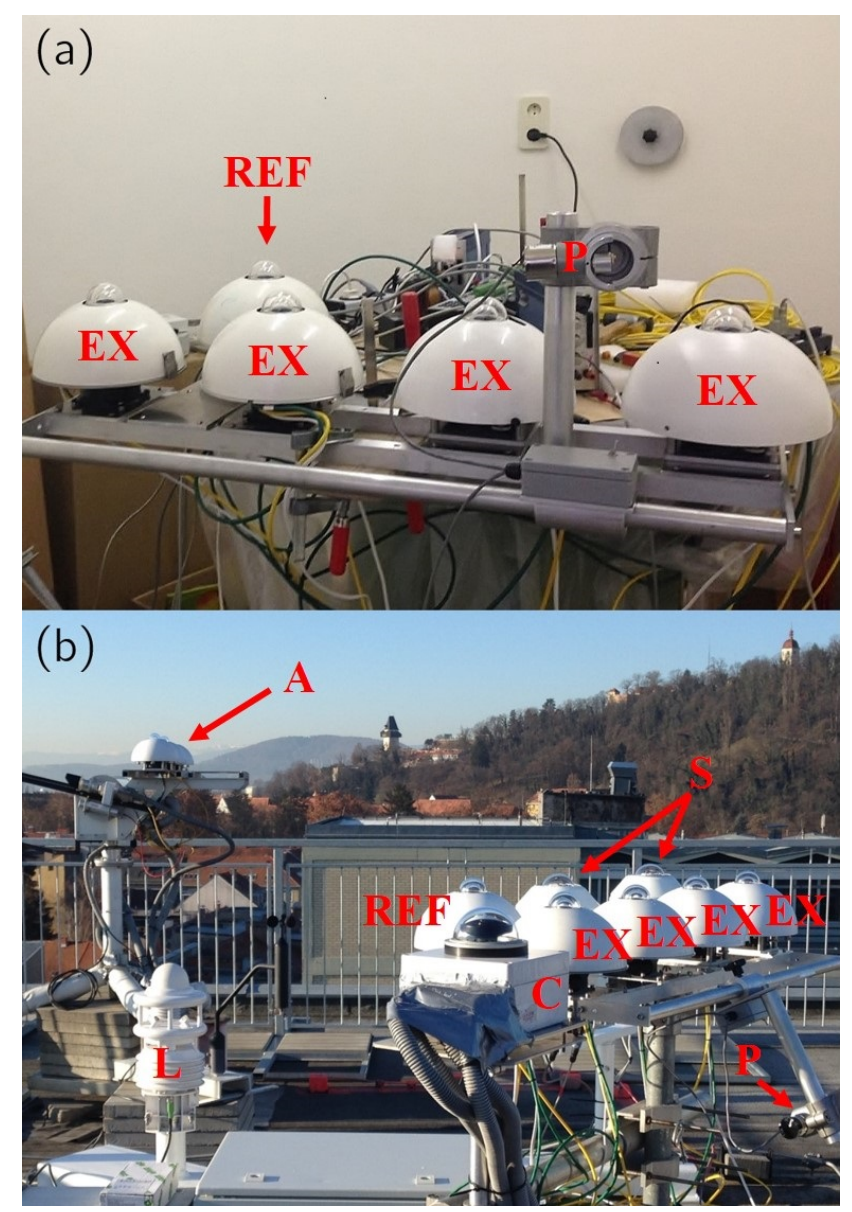

Figure 2. Measurement setup at (a) the Kanzelhöhe Observatory and (b) the measurement platform of Graz University in the direct vicinity of the ARAD site. During the field campaigns the measurement setup was expanded by an "all-in-one" meteorological observing system for the monitoring of ambient meteorological conditions, two star pyranometers, and a cloudcam. The labels are defined as follows: REF is the reference CMP21 pyranometer contained in an Eigenbrodt SBL $480 \mathrm{HV}$-system; EX are the "experimental" CMP21 pyranometers contained in an Eigenbrodt SBL 480 HV-system, a Davos (PMOD/WRC) HV-system, a self-built KSO34 $\mathrm{HV}$-system, and another self-built KSO HV-system; P is the electric motor pump used for the automated spray tests; L is the all-inone meteorological observing system (WS600-UMB manufactured by Lufft $\mathrm{GmbH}$ ); $\mathrm{C}$ is the cloudcam (VIS-J1006, manufactured by CMS Schreder GmbH); S are two star pyranometers (type 8102, manufactured by Schenk); and A is the Graz University ARAD site.

an overview of the measurement setup in the laboratory of the Kanzelhöhe Observatory.

Following the laboratory experiments, two field campaigns (18 to 25 January 2016 and 12 April to 3 May 2016) were performed. Figure $2 \mathrm{~b}$ shows the measurement setup during the field campaigns in the direct vicinity of the Graz University ARAD site.
During laboratory and field campaigns for each pyranometer/HV-system combination, a series of eight controlled spray tests was performed.

\section{Results}

\subsection{Laboratory experiments}

\subsubsection{Comparison of pyranometers under undisturbed conditions}

The initial investigations of the laboratory campaign were centered on the comparison of CMP21 performance when operated within the different HV-systems used within the ARAD network. To this aim, pyranometer output was compared during dormant phases (without external impact factors). This comparison focused on (i) the temporal stability of pyranometer/HV-system combinations when operated in a steady environment and (ii) the differences in the absolute values of the pyranometer outputs when operated in different HV-systems under the same controlled ambient conditions. The differences and spread in pyranometer output values were established over a measurement interval of $65 \mathrm{~h}$, following a $24 \mathrm{~h}$ system spin-up phase, and are summarized in Fig. 3. Under controlled laboratory conditions, differences among the CMP21 pyranometers operated in the same HVsystem were on average smaller than $1 \mathrm{~W} \mathrm{~m}^{-2}$, and the output values of CMP21 pyranometers across the $\mathrm{HV}$-systems were within $\pm 1 \mathrm{~W} \mathrm{~m}^{-2}$.

Given the general stable performance of pyranometers within each $\mathrm{HV}$-system and the small differences in output values (we note that most of the ARAD sites resolve pyranometer output at coarser resolution than during experiments, i.e., $1 \mathrm{~W} \mathrm{~m}^{-2}$ steps), an Eigenbrodt SBL $480 \mathrm{HV}$ system was used as housing for the undisturbed reference pyranometer (REF) during all experiments (laboratory and field), as it is also the most frequently used HV-system within the ARAD network (Olefs et al., 2016).

\subsubsection{Spray tests under controlled conditions}

After the initial instrument comparison, a series of spray tests was performed for each pyranometer/HV-system combination.

The experiments comprised eight spray tests per pyranometer/HV-system combination, each with 30 strokes and a $3 \mathrm{~h}$ dormant phase between individual spray tests to allow the systems to recover to the initial state. The amount of water applied per spray test was approximately $3.4 \mathrm{~mL}$. In the following, the experimental pyranometer/HV-system combinations are referred to as $\mathrm{EIG}_{\mathrm{EX}}, \mathrm{KSO} 4_{\mathrm{EX}}$, and $\mathrm{DAV}_{\mathrm{EX}}$, respectively.

Figure 4a provides a time series of one of the spray tests performed during the laboratory campaign. The output signal of KSO34 $4_{\mathrm{EX}}$ decreased by approximately the same value 


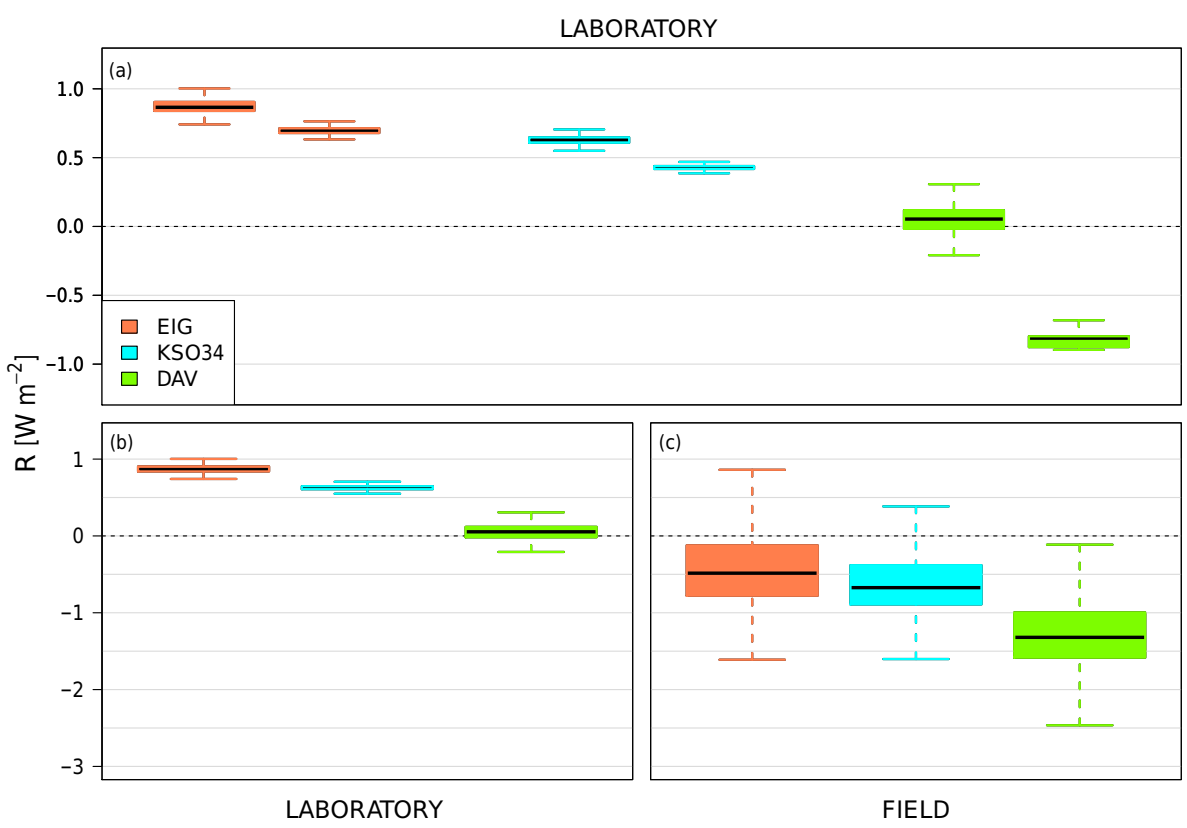

Figure 3. Spread of radiation $(R)$ measurements with CMP21 pyranometers contained in different HV-systems (EIG, KSO34, and DAV) during dormant (i.e., undisturbed) phases for (a) and (b), laboratory, and for (c), field campaigns. Panel (a) provides results for two CMP21 pyranometers per $\mathrm{HV}$-system as multiple instruments were available during the laboratory campaign.
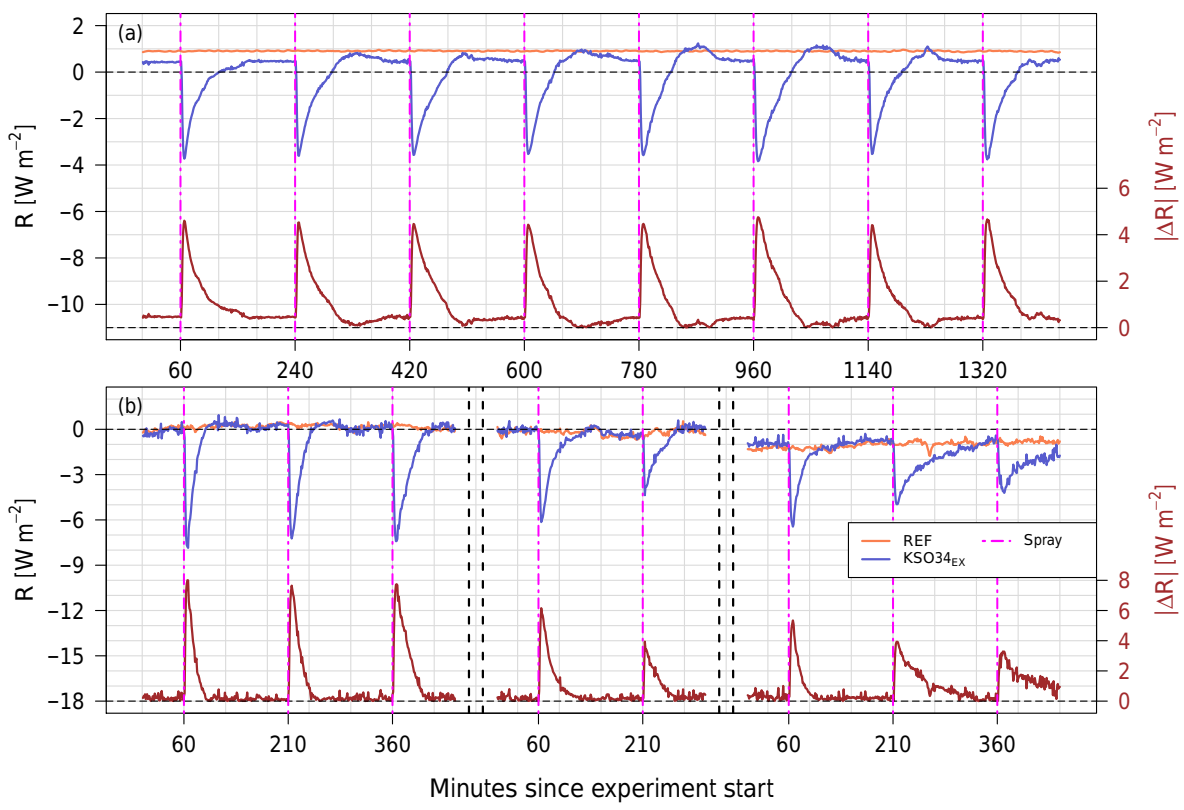

Figure 4. Time series of the radiation $(R)$ measured by the reference (REF, coral, Eigenbrodt HV-system) and experimental pyranometer (KSO34 ${ }_{\mathrm{EX}}$, blue, KSO HV-system) and the absolute difference $(|\Delta R|$ ) between REF and KSO34 $\mathrm{EX}$ during (a) laboratory conditions and (b) ambient environmental conditions. All field measurements were performed during nighttime. The measurement series is continuous in (a), while the start point of subpanels (separated by vertical double dashed lines) in (b) is always 18:30 UTC. Note that the scales of the $y$ axes differ between panels. 


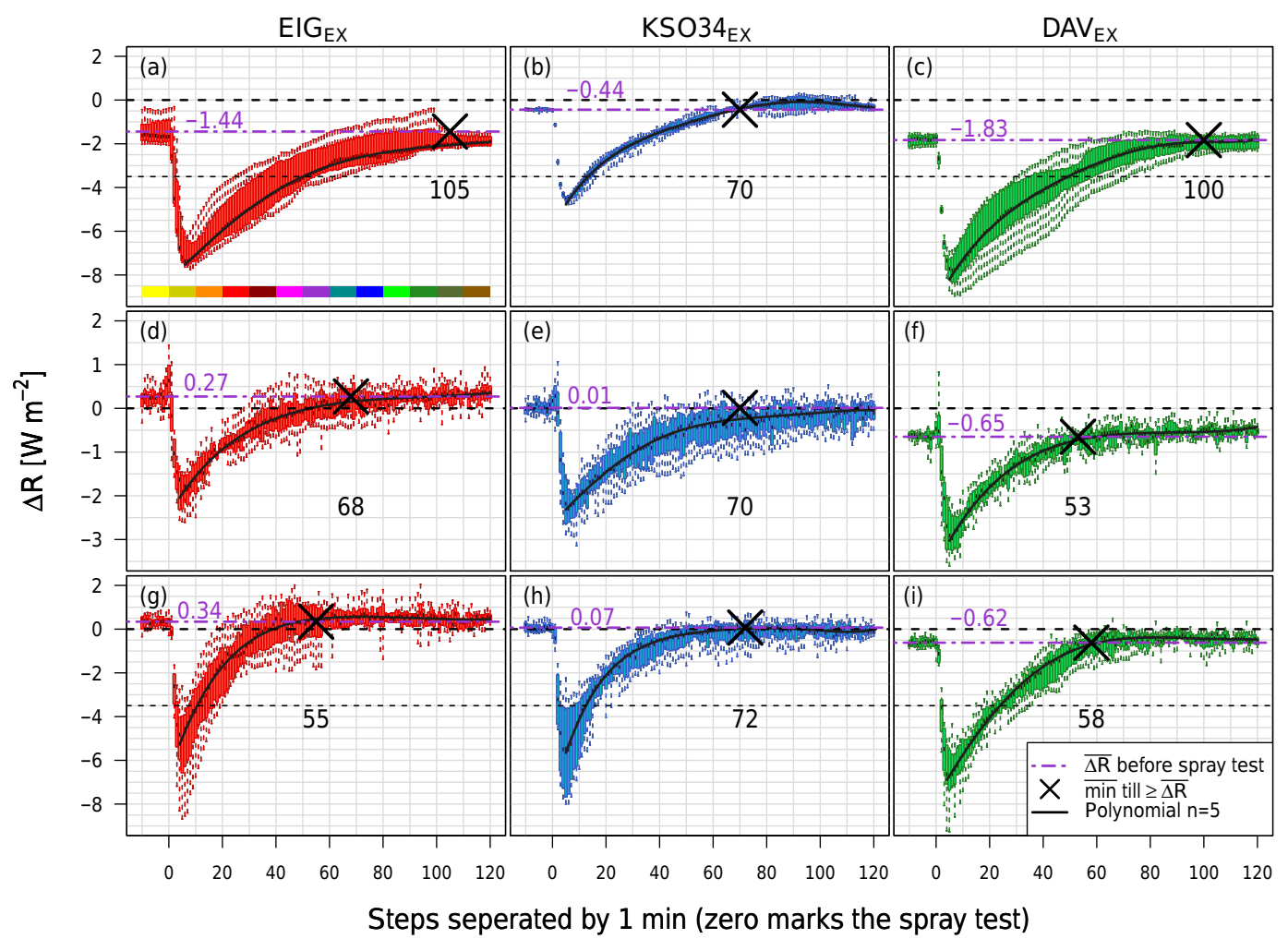

Figure 5. Box-whisker plots (in one minute time intervals) of the difference in radiation measurements $(\Delta R)$ between individual experimental pyranometer/HV-system combinations $\left(\mathrm{EIG}_{\mathrm{EX}}\right.$ in red, $\mathrm{KSO} 34_{\mathrm{EX}}$, in blue and $\mathrm{DAV} \mathrm{EX}_{\mathrm{EX}}$ in green) and the reference pyranometer (REF) following the spray tests. Panels (a)-(c) show the results from the experiments during the laboratory campaign, panels (d)-(f) show the results from the first field campaign (January 2016), and panels (g)-(i) show the results from the second field campaign (April-May 2016). The $x$ axis in all panels shows the experiment time, starting $10 \mathrm{~min}$ before and ending $120 \mathrm{~min}$ after the spray tests (marked with zero). The purple dot-dashed horizontal line marks the average difference $\overline{\Delta R}$ before the spray test. The black cross marks the average time in minutes which each pyranometer/HV-system pair needed to return to or exceed its initial state (numbers give corresponding time in minutes). The color bar in panel (a) represents the temporal evolution of experiments further analyzed in Fig. 7. In all panels, a polynomial of 5th degree (black solid line) of the median values, beginning at the minimum (maximum pyranometer response), is shown. Note that the scales of the $y$ axes in (d)-(f) differ from those in (a)-(c) and (g)-(i).

$\left(\sim-4 \mathrm{~W} \mathrm{~m}^{-2}\right)$ during each experiment and needed about $1-$ $2 \mathrm{~h}$ to recover to its initial state thereafter. Similar results were obtained for other pyranometer/HV-system combina-

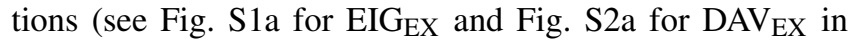
the Supplement to this article). The pyranometer response to spray tests is attributed to a change in the thermal balance, i.e., the outer glass dome experiences evaporative cooling during and following the spray test.

Figure 5a-c show box-whisker plots of average pyranometer responses for the period spanning $10 \mathrm{~min}$ before to $120 \mathrm{~min}$ after the spray tests (marked with zero) for $\mathrm{EIG}_{\mathrm{EX}}, \mathrm{KSO} 4_{\mathrm{EX}}$, and DAV $\mathrm{EX}_{\mathrm{EX}}$. The purple dot-dashed horizontal line marks the average difference $\overline{\Delta R}$ before the spray test, and the black cross marks the average time in minutes which each pyranometer/HV-system needed to return to or exceed its initial state. Zero offsets exceeded $-4 \mathrm{~W} \mathrm{~m}^{-2}$ for all pyranometer/HV-system combinations, and offsets as large as $\sim-8.5 \mathrm{~W} \mathrm{~m}^{-2}$ were observed. The recovery time to the initial state following the spray tests ranged among pyranometer/HV-system combinations between $70 \mathrm{~min}\left(\mathrm{KSO} 4_{\mathrm{EX}}\right)$ and $105 \mathrm{~min}\left(\mathrm{EIG}_{\mathrm{EX}}\right)$.

The results from these laboratory experiments confirm the substantial influence of precipitation events on pyranometer measurements as observed during routine observations in the ARAD network. Furthermore, significant zero offsets occur independently of the HV-system used and recovery to initial state exceeds $1 \mathrm{~h}$ throughout. These results motivated a series of experiments under the ambient environmental conditions described below, directed towards a better understanding of the magnitude of pyranometer offsets due to precipitation events.

\subsection{Field experiments}

Following the laboratory experiments two, extensive field campaigns were performed in January and April-May 2016. During these campaigns, parallel measurements with a se- 
ries of CMP21 pyranometers were performed in the direct vicinity of the Graz University ARAD site. The measurement setup comprised one reference pyranometer (REF, contained in EIG) and three experimental pyranometers (EX, contained in EIG, KSO34, and DAV HV-systems). Additionally, ambient meteorological conditions (air temperature, air humidity, wind direction, and wind speed) were monitored with an "all-in-one" meteorological observing system, WS600UMB manufactured by Lufft $\mathrm{GmbH}$, hereinafter referred to as LUFFT.

As our main interest lies in studying pyranometer zerooffsets and the effect of precipitation events, the majority of experiments were performed during nighttime conditions. When ambient environmental conditions allowed (no natural precipitation), three experiments were performed per night with a $2.5 \mathrm{~h}$ dormant phase between individual experiments. The dormant phase was reduced by 30 min compared to laboratory experiments following the initial result of pyranometer signal recovery to initial state after the laboratory spray tests. Naturally, nighttime conditions are less relevant to radiation monitoring, though they provide a natural reference framework for the determination of instrument offsets. Furthermore, any type of lens effect due to drop formation following precipitation events can be ruled out during nighttime conditions. Because of the potential relevance for estimating the effect of precipitation events on radiation monitoring accuracy during a routine daytime operation, an additional series of spray tests was performed under daylight conditions.

\subsubsection{Comparison of pyranometers under undisturbed conditions}

First we turn the focus to the comparison of pyranometers contained in different $\mathrm{HV}$-systems under ambient, undisturbed, and nighttime conditions. Figure $3 \mathrm{c}$ summarizes the results for both field campaigns. Comparison with laboratory experiments (Fig. 3a and b) show that the range of pyranometer output increases during ambient conditions reaching values of up to $2.4 \mathrm{~W} \mathrm{~m}^{-2}$. Nevertheless, the median difference in radiation measurements $\widetilde{\Delta R}$ between laboratory (Fig. 3b) and field (Fig. 3c) conditions yields very similar results for individual pyranometer/HV-system pairs: $\left|\widetilde{\Delta R}_{\mathrm{EIG}}\right|=1.35 \mathrm{~W} \mathrm{~m}^{-2}, \quad\left|\widehat{\Delta R}_{\mathrm{KSO} 34}\right|=1.30 \mathrm{~W} \mathrm{~m}^{-2}$, and $\left|\widetilde{\Delta R}_{\mathrm{DAV}}\right|=1.37 \mathrm{~W} \mathrm{~m}^{-2}$.

\subsubsection{Spray tests during nighttime}

Following the initial state comparison, a series of spray tests was performed for each pyranometer/HV-system combination under ambient environmental conditions. The automated spray tests were performed for one system at a time, i.e., while one system was sprayed, the reference system and all other experimental systems remained undisturbed. Figure $4 \mathrm{~b}$ shows a time series of pyranometer measurements during spray tests performed with a CMP21 pyranometer contained in a KSO34 HV-system. The output signal of KSO34 EX decreased by $4 \mathrm{~W} \mathrm{~m}^{-2}$ (or more) during each experiment, a result very similar to experiments under laboratory conditions (Fig. 4a). This also holds true for other pyranometer/HVsystem combinations, see Fig. S1b (EIGEX) and Fig. S2b $\left(\mathrm{DAV}_{\mathrm{EX}}\right)$ in the Supplement.

The response of pyranometer/HV-system combinations to spray tests under ambient environmental conditions is further explored in panels (d)-(f) (first field campaign, January 2016) and in panels (g)-(i) (second field campaign, AprilMay 2016) of Fig. 5. Here, pairwise differences between experimental systems and REF during spray tests under ambient environmental conditions are shown. Each comparison combines the measurements of eight spray tests, starting $10 \mathrm{~min}$ before and ending $120 \mathrm{~min}$ after each spray test.

Independent of the HV-system used, all pyranometers reacted immediately to spray tests and reached their maximum response (minimum value) within $5 \mathrm{~min}$ after the test. Overall responses are, in magnitude, similar among evaluated systems and comparable to laboratory results.

Nevertheless, the time period needed by pyranometers/HV-systems to recover to their initial states varied among the instruments. Under laboratory conditions, the average time needed to recover is similar for $\mathrm{EIG}_{\mathrm{EX}}$ and $\mathrm{DAV}_{\mathrm{EX}}$, while pyranometers contained in KSO34 $\mathrm{EX}$ recover significantly faster. Under ambient environmental conditions, the recovery times of EIG $\mathrm{EX}_{\text {and }} \mathrm{DAV} \mathrm{EX}_{\mathrm{EX}}$ are 55 and $58 \mathrm{~min}$ respectively, which are approximately half their respective laboratory values $\left(\mathrm{EIG}_{\mathrm{EX}}\right.$ : $105 \mathrm{~min}$ and $\mathrm{DAV}_{\mathrm{EX}}: 100 \mathrm{~min}$ ), while the recovery times for KSO34 $\mathrm{EX}$ are not significantly different during ambient and laboratory conditions. Overall, the results indicate a faster recovery of the pyranometer response under ambient environmental conditions (attributed mainly to enhanced drying due to wind and ambient air temperature), in contrast to laboratory conditions. Nevertheless, all pyranometers required substantial time (at least $53 \mathrm{~min}$ ) to return to their initial signal states after the simulated precipitation events.

An interesting aspect is the relationship between pyranometer response and ambient air temperature $\left(T_{\mathrm{a}}\right)$, as air temperature increases the variance in pyranometer response to spray tests. The influence of $T_{\mathrm{a}}$ is directly linked to downward longwave radiation $\left(L_{\text {down }}\right)$, which governs $T_{\mathrm{a}}$. While during undisturbed conditions a moderate relationship between pyranometer offsets and $L_{\text {down }}$ and $T_{\mathrm{a}}$ is found, precipitation largely overwhelms infrared effects. Figure 6 illustrates this almost linear relationship (i.e., the larger $L_{\text {down }}$ and higher $T_{\mathrm{a}}$, the larger the pyranometer response following a (simulated) precipitation event) for the results of the AprilMay field campaign. Panels (a) and (e) show scatterplots of $L_{\text {down }}$ and $R$ and $T_{\mathrm{a}}$ and $R$, respectively, for the time interval 15-5 min prior to the spray tests. Conversely, panels (d) and (h) show the same relationship for the interval 105-115 min following the spray tests. Immediately following the spray tests, a significantly larger system response emerges leading 


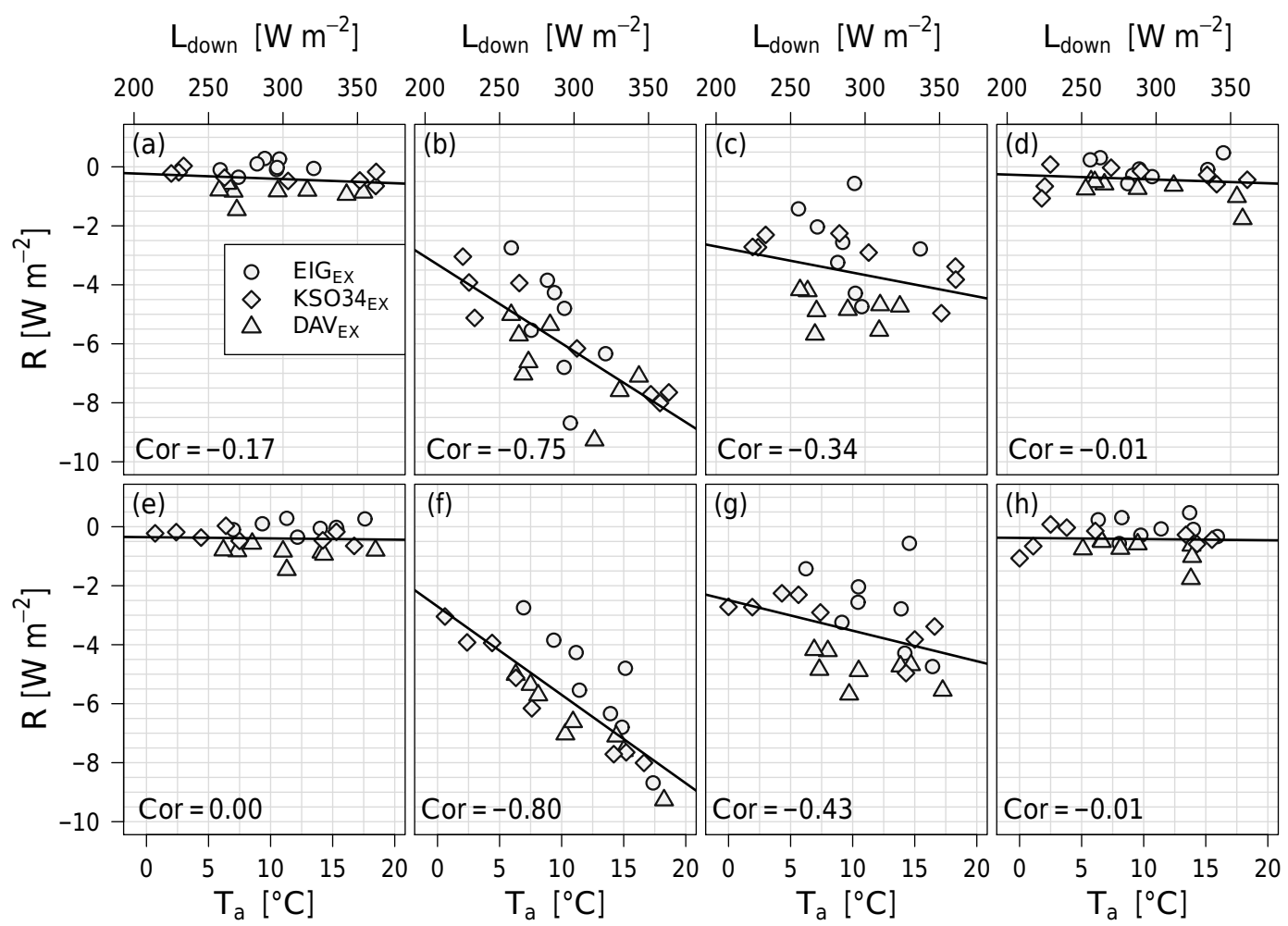

Figure 6. Minimum radiation measurements (maximum pyranometer responses) during nighttime as function of (a)-(d) downward longwave radiation $\left(L_{\text {down }}\right.$, from Graz University ARAD site) and (e)-(h) ambient air temperature $\left(T_{\mathrm{a}}\right.$, measured by LUFFT) during the field campaign in April-May 2016. Individual panels show minimum values of $R$ and $L_{\text {down }}$ for the selected time intervals: (a) 15-5 min before spray tests, (b) within 0-5 min after spray tests, (c) 15-25 min after spray tests, and (d) 105-115 min after spray tests. (e) -(h) as (a)-(d) but for the comparison of $R$ and $T_{\mathrm{a}}$. Spearman's rank correlation coefficient (Cor) is provided in each panel.

to a stronger connection with $L_{\text {down }}$ and $T_{\mathrm{a}}$ (see panels b and f), which is reduced over time as the system recovers towards its initial state (see panels (c) and (g) for the time interval 15-25 min after the spray tests). The relationship between pyranometer response and ambient air temperature also becomes visible when comparing the results of the April-May (Fig. 5g-i) and January field campaigns (Fig. 5d-f).

Since the standard setup for spray tests during the field campaigns did not allow for a one-to-one comparison of pyranometer responses during the spray tests, an additional set of experiments was performed where individual experimental pyranometers were sprayed in series under the same ambient environmental conditions. Figure 7 shows a one-toone comparison of the consecutively sprayed instruments, i.e., $\mathrm{DAV}_{\mathrm{EX}}$ as a function of $\mathrm{EIG}_{\mathrm{EX}}$ and $\mathrm{KSO} 4_{\mathrm{EX}}$ as a function of $\mathrm{DAV}_{\mathrm{EX}}$. The colors mark bins (size $10 \mathrm{~min}$ ) of measurements ranging from $10 \mathrm{~min}$ before (yellow) to $120 \mathrm{~min}$ after (brown) the spray tests (see color bar in Fig. 5a for the temporal evolution of experiments). The results show a good agreement among individual system responses, with an explained variance (squared Spearman's rank correlation coefficient) of Var $=0.68$ between $\mathrm{EIG}_{\mathrm{EX}}$ and $\mathrm{DAV}_{\mathrm{EX}}$ and $\mathrm{Var}=0.66$ between $\mathrm{DAV}_{\mathrm{EX}}$ and $\mathrm{KSO} 4_{\mathrm{EX}}$.

\subsubsection{Spray tests during daytime}

Having established pyranometer responses to simulated precipitation events, our focus shifted to the analysis of instrument responses under ambient daytime conditions. To this aim, a series of spray tests was performed for each pyranometer/HV-system pair at the end of the second field campaign. The panels in Fig. 8 illustrate one test each for the pyranometers contained in the three considered HVsystems. As expected, pyranometer responses are larger during daytime conditions, reaching differences to REF of up to $-100 \mathrm{Wm}^{-2}$. The time needed for the sensors to recover to the initial states was significantly shorter than during nighttime conditions $\left(\mathrm{EIG}_{\mathrm{EX}}, 9 \mathrm{~min} ; \mathrm{KSO} 34_{\mathrm{EX}}, 7 \mathrm{~min}\right.$; and $\mathrm{DAV}_{\mathrm{EX}}, 25 \mathrm{~min}$ ), indicating rapid sensor adjustment. The larger system response but shorter recovery time indicates that recovery depends strongly on evaporation - i.e., the stronger the evaporation of the droplets on the glass dome, due to ambient temperature and wind speed, the smaller the time a pyranometer needs to recover to its initial state. A reasonable explanation considering evaporation depends on the radiation budget, temperature, relative humidity, and wind (Kraus, 2004). 

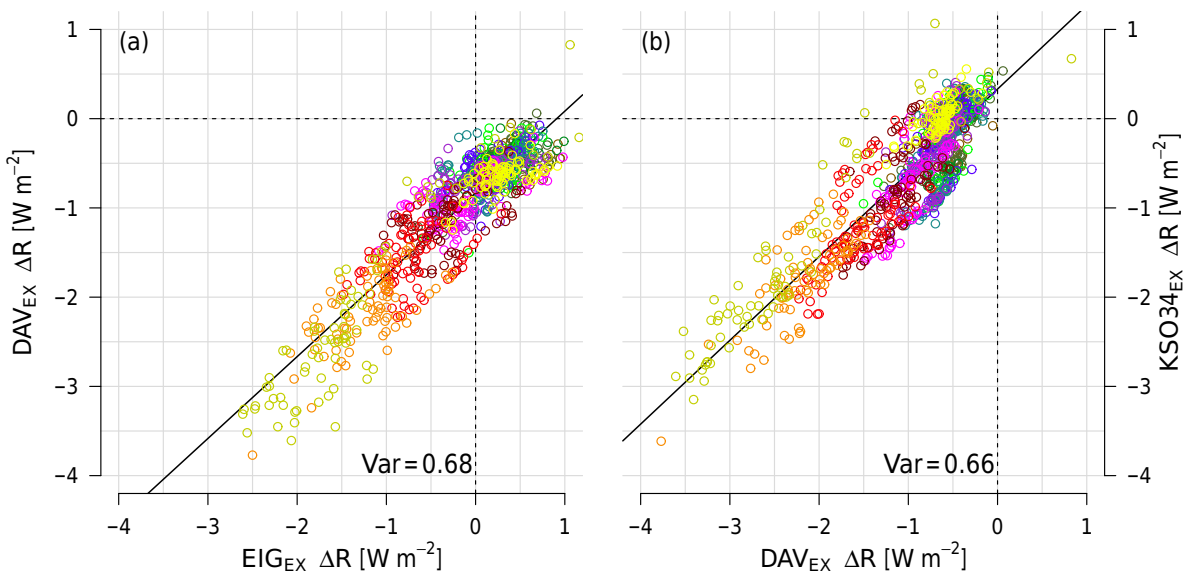

Figure 7. Scatter plots of the difference in radiation $(\Delta R)$ between the individual experimental pyranometer/HV-system combinations $\left(\mathrm{EIG}_{\mathrm{EX}}, \mathrm{KSO} 4_{\mathrm{EX}}\right.$, and $\left.\mathrm{DAV}_{\mathrm{EX}}\right)$ and the reference pyranometer (REF) during nighttime. In both panels, the pyranometer sprayed second is given as a function of the one sprayed first. The colors mark bins (size 10 min with 1 min resolution) of measurements ranging from $10 \mathrm{~min}$ before (yellow) to $120 \mathrm{~min}$ after (brown) the spray tests (see color bar in Fig. 5a for temporal evolution of experiments). The relationship between two pyranometers is characterized by the squared Spearman's rank correlation coefficient (Var).

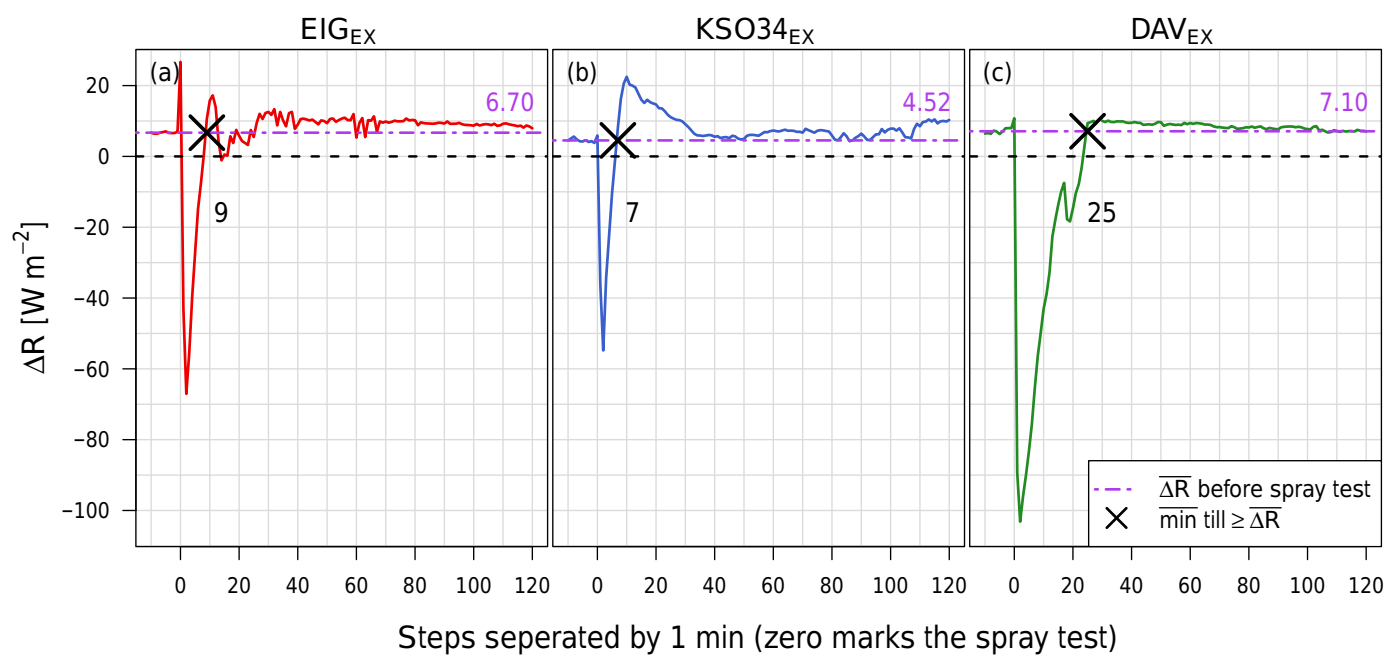

Figure 8. Difference in radiation $(\Delta R)$ between the individual experimental pyranometer/HV-system combinations and the reference pyra-

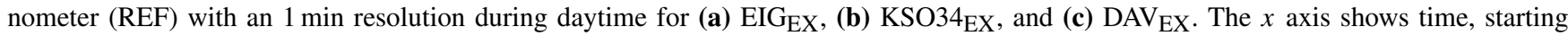
$10 \mathrm{~min}$ before and ending $120 \mathrm{~min}$ after the spray tests (marked with zero). The purple dot-dashed horizontal line marks the average difference $\overline{\Delta R}$ before the spray test, and the black cross marks the average time in minutes which each pyranometer/HV-system combination needed to return to or exceed its initial state. Note that these experiments were not performed in parallel for technical reasons.

\section{Discussion and conclusions}

This study seeks to investigate the influence of instrumentation and precipitation events on the accuracy of radiation measurements within the Austrian radiation monitoring network. To this aim, one laboratory and two field campaigns were performed in 2016, investigating pyranometer performance in different heating and ventilation systems (HVsystems) as well as zero offsets triggered by precipitation events. During the campaigns, pyranometers of type CMP21 (Kipp\&Zonen) were operated as "experimental" in three different HV-systems (Eigenbrodt SBL $480 \mathrm{EIG}_{\mathrm{EX}}$, Davos (PMOD/WRC) $\mathrm{DAV}_{\mathrm{EX}}$, and the self-built $\mathrm{KSO} 4_{\mathrm{EX}}$ ) and measurements were compared with the output of an undisturbed reference pyranometer (CMP21, contained in a housing of type Eigenbrodt SBL 480). To determine the effect of precipitation on measurement accuracy a series of more than 115 simulated precipitation events (as standardized spray tests) was performed.

The first campaign (January 2016) comprised a series of laboratory experiments at the Kanzelhöhe Observatory. The results of the campaign showed that (i) under undis- 
turbed dormant conditions, pyranometer output values lie within $\pm 1 \mathrm{~W} \mathrm{~m}^{-2}$, independent of the HV-system; (ii) standardized spray tests $(3.4 \mathrm{~mL}$ of distilled water) trigger zero offsets of $-4 \mathrm{~W} \mathrm{~m}^{-2}$ or more; and (iii) the time individual pyranometer/HV-system combinations needed to recover to initial states after spray tests differed but exceeded $70 \mathrm{~min}$ for all systems. The pronounced pyranometer response following spray tests is attributed to a change in the thermal balance, i.e., the evaporative cooling of the outer glass dome.

Following the laboratory campaign, two intensive field campaigns were performed in January and April-May 2016 in the direct vicinity of the Graz University ARAD site at the measurement platform of the University of Graz. During the field campaigns, the same setup for radiation measurements (three experimental and one reference pyranometer) was used as during the laboratory campaign. Additionally, ambient meteorological conditions (air temperature, air humidity, precipitation, wind speed, and direction) were monitored with an all-in-one meteorological observing system (LUFFT).

The results of the field campaign showed that (i) the range of pyranometer output increased during ambient nighttime conditions, reaching values of up to $2.4 \mathrm{~W} \mathrm{~m}^{-2}$; (ii) all pyranometers reacted immediately to spray tests and reached their maximum response (minimum value) within $5 \mathrm{~min}$ of the test; (iii) pyranometer responses were similar among evaluated systems and comparable to laboratory results; and (iv) individual pyranometer/HV-system combinations recovered faster to their initial states following spray tests under ambient environmental conditions, which is mainly attributed to enhanced drying due to wind and ambient air temperature. Furthermore, a quasilinear relationship between the strength of the pyranometer response (decrease) after a spray test and downward longwave radiation and ambient air temperature was found.

An additional set of experiments performed during daylight conditions indicated a significant effect of precipitation events during routine radiation monitoring. Differences of the experimental pyranometers to the undisturbed reference system reached up to $-100 \mathrm{Wm}^{-2}$ and sensors recovered substantially faster (within a few minutes) to initial states than during nighttime conditions, which is attributed to evaporation effects.

In summary, the results from the series of laboratory and field experiments show a stable and comparable performance of CMP21 pyranometers throughout the different HV-systems used within the ARAD network. A significant effect of precipitation on the accuracy of daytime radiation measurements and nighttime zero offsets was found independent of the pyranometer/HV-system combination. The substantial time individual systems need to recover to stable initial states after precipitation events motivates flagging recommendations for operational use in the ARAD network. Precipitation data are available at all ARAD sites from colocated meteorological stations. We recommend flagging ra- diation measurements during and after precipitation events as system stability is not ensured, as our results show. We recommend (i) the flagging of daytime radiation measurements as "wrong" during precipitation events and "dubious" for 10 min following precipitation events, and (ii) the flagging of nighttime outputs as wrong during precipitation events and dubious for $90 \mathrm{~min}$ following precipitation events. Furthermore, we recommend applying the same flagging criteria and intervals of the precipitation events for routine pyranometer cleaning if water or alcohol is sprayed on the pyranometer's outer glass dome. Similar flagging criteria might be useful to also improve metadata information in other radiation monitoring networks.

We note in closing, that additional field and/or laboratory experiments characterizing pyranometer offsets following abrupt temperature changes and for different precipitation types (e.g., snow, freezing rain, rain and snow mixes) would strongly increase our understanding of the influence of ambient meteorology, and the abrupt changes therein, on the stability and measurement accuracy of BSRN-class pyranometers in different HV-systems. Further additional analyses regarding offsets following precipitation events for unventilated pyranometers are recommended.

Data availability. Data presented in this article are available at https://www.kso.ac.at/publication_data/oswald_amt_2017/ (Oswald, 2017).

\section{The Supplement related to this article is available online at doi:10.5194/amt-10-1169-2017-supplement.}

Competing interests. The authors declare that they have no conflict of interest.

Acknowledgements. The authors thank the Austrian Zentralanstalt für Meteorologie und Geodynamik (ZAMG) for providing CMP21 pyranometers, the Eigenbrodt SBL 480, and the Davos (PMOD/WRC) HV-systems operated during campaigns as well as the data from the Graz University TAWES site and the Graz University ARAD site. The authors are grateful to Martin Mair (ZAMG) for technical support and fruitful discussions, Erich MurschRadlgruber (University of Natural Resources and Life Sciences, BOKU, Vienna) for providing an all-in-one meteorological observing system (WS600-UMB by Lufft GmbH), and Heinrich Freislich (Kanzelhöhe Observatory) for technical support and constructions. S. Oswald acknowledges financial support through fellowships from the Department of Environmental, Regional, and Educational Sciences of the University of Graz and Grazer Wechselseitige Versicherungen AG. The authors acknowledge the financial support by the University of Graz. The authors thank Joseph Michalsky and an anonymous referee for their helpful comments during the discussion phase of this article. 
Edited by: S. Malinowski

Reviewed by: J. Michalsky and one anonymous referee

\section{References}

Augustine, J. A. and Dutton, E. G.: Variability of the surface radiation budget over the United States from 1996 through 2011 from high-quality measurements, J. Geophys. Res.-Atmos., 118, 43-53, doi:10.1029/2012jd018551, 2013.

Dutton, E. G., Stone, R. S., Nelson, D. W., and Mendonca, B. G.: Recent Interannual Variations in Solar Radiation, Cloudiness, and Surface Temperature at the South Pole, J. Climate, 4, 848858, doi:10.1175/1520-0442(1991)004<0848:rivisr>2.0.co;2, 1991.

Gilgen, H., Wild, M., and Ohmura, A.: Means and trends of shortwave incoming radiation at the surface estimated from Global Energy Balance Archive data, J. Climate, 11, 2042-2061, 1998.

Kipp and Zonen: CMA series CMP series Instruction Manual Version 1007, Delft (Netherlands), 1, 2010.

Kraus, H.: Die Atmosphäre der Erde, Springer-Verlag Berlin Heidelberg, doi:10.1007/3-540-35017-9, 2004.

Liepert, B. G.: Observed reductions of surface solar radiation at sites in the United States and worldwide from 1961 to 1990 , Geophys. Res. Lett., 29, 61-1-61-4, doi:10.1029/2002gl014910, 2002.

McArthur, B. L. J.: Baseline Surface Radiation Network (BSRN). Operations Manual. Version 2.1, WCRP-121, available at: http: //hdl.handle.net/10013/epic.39582.d001 (last access: December 2016), 2005.

Ohmura, A., Gilgen, H., Hegner, H., Müller, G., Wild, M., Dutton, E. G., Forgan, B., Fröhlich, C., Philipona, R., Heimo, A., König-Langlo, G., McArthur, B., Pinker, R., Whitlock, C. H., and Dehne, K.: Baseline Surface Radiation Network (BSRN/WCRP): New Precision Radiometry for Climate Research, B. Am. Meteor. Soc., 79, 2115-2136, doi:10.1175/15200477(1998)079<2115:bsrnbw>2.0.co;2, 1998.
Olefs, M., Baumgartner, D. J., Obleitner, F., Bichler, C., Foelsche, U., Pietsch, H., Rieder, H. E., Weihs, P., Geyer, F., Haiden, T., and Schöner, W.: The Austrian radiation monitoring network ARAD - best practice and added value, Atmos. Meas. Tech., 9, 15131531, doi:10.5194/amt-9-1513-2016, 2016.

Oswald, S. M.: Data set to "Pyranometer offsets triggered by ambient meteorology: insights from laboratory and field experiments", available at: https://www.kso.ac.at/publication_data/ oswald_amt_2017/, 2017.

Ramanathan, V.: The role of earth radiation budget studies in climate and general circulation research, J. Geophys. Res., 92, 4075, doi:10.1029/jd092id04p04075, 1987.

Stanhill, G.: Global dimming: A new aspect of climate change, wea, 60, 11-14, doi:10.1256/wea.210.03, 2005.

Wild, M.: From Dimming to Brightening: Decadal Changes in Solar Radiation at Earth's Surface, Science, 308, 847-850, doi:10.1126/science.1103215, 2005.

Wild, M.: Global dimming and brightening: A review, J. Geophys. Res., 114, doi:10.1029/2008jd011470, 2009.

Wild, M., Folini, D., Schär, C., Loeb, N., Dutton, E. G., and KönigLanglo, G.: The global energy balance from a surface perspective, Clim. Dyn., 40, 3107-3134, doi:10.1007/s00382-012-15698, 2012.

Wild, M., Folini, D., Hakuba, M. Z., Schär, C., Seneviratne, S. I., Kato, S., Rutan, D., Ammann, C., Wood, E. F., and KönigLanglo, G.: The energy balance over land and oceans: an assessment based on direct observations and CMIP5 climate models, Clim. Dyn., 44, 3393-3429, doi:10.1007/s00382-014-2430z, 2014. 